\title{
The RAMOD astrometric observable and the relativistic astrometric catalogs
}

\author{
M. T. Crosta ${ }^{1}$, B. Bucciarelli ${ }^{1}$, F. de Felice ${ }^{2}$, M. G. Lattanzi ${ }^{1}$ \\ and A. Vecchiato ${ }^{1}$ \\ ${ }^{1}$ INAF-OATo, Strada Osservatorio 20, Pino Torinese, Torino, Italy \\ ${ }^{2}$ University "G.Galileo", Dept. of Physics, via Marzolo 8, Padova, Italy
}

\begin{abstract}
We describe a way to compare current relativistic astrometric models accurate to the micro-arcsecond level. The observed stellar direction can be written as a function of several parts, linking the astrometric observables to the relativistic effects associated to the stellar kinematical properties and distances as seen inside the gravitational field of our Solar System, i.e. the so called relativistic astrometric parameters, providing a tool for comparing the RAMOD framework to the $\mathrm{pM} / \mathrm{pN}$ approaches.
\end{abstract}

Keywords. astrometry, general relativity, solar system: general, catalogs

\section{Relativistic astrometric models}

It has been shown (Kopeikin and Mashhoon 2002; Klioner 2003; de Felice et al. 2006 and references therein) that, at the micro-arcsecond level of accuracy, astrometry needs General Relativity (GR) to trace back the stellar position and take into account all the effects due to the gravitating bodies and their tidal stresses on the background geometry at the same level of accuracy. There are three main approaches to deal with the problem of the relativistic sphere reconstruction. But ways to implement the current astrometric models in the context of Gaia mission and optimal strategies for their detailed comparisons should still be devised. This contribution goes toward this effort. (i) As a Parameterized Post Newtonian (PPN) extension of a seminal study conducted in the framework of post-Newtonian (pN) approximation of GR (Klioner \& Kopeikin, 1992), Klioner (2003) produced a PPN formulation of a model for relativistic astrometry, accurate to $1 \mu \mathrm{as}$, in which the finite distance and the angular momentum of the gravitational deflector are included, linked to the motion of the observer and the source in order to consider the effects of parallax, aberration, and proper motion. This model is considered the baseline for the Gaia data reduction (GREM). (ii) Kopeikin \& Schäfer (1999), using the pM approximation, solved the metric tensor in retarded Lienard-Weichert potentials and later Kopeikin \& Mashhoon (2002) included all relativistic effects related to the gravitomagnetic field, produced by the translational velocity/spin-dependent metric terms. Both studies rewrite the null geodesic as a function of two independent parameters and solve the light trajectory as a straight line (Euclidean geometry) plus corrections in the form of integrals, containing the perturbations encountered, from a source at an arbitrary distance to an observer located within the Solar System. (iii) The same parameterization can be obtained in RAMOD3 (de Felice et al., 2004), where it is always possible to map the null geodesic onto hypersurfaces of simultaneity with the epoch of observation (Crosta, 2003). RAMOD is a well-established framework of general relativistic astrometric models which can be extended to any accuracy and physical requirements. It tries to be as close as possible to the concept of curved geometry of GR in order to reconstruct all simultaneous observations in a curved spacetime (de Felice \& Clarke 1990). Recent 
developments have included the retarded distance effects, due to the moving bodies of solar system, in the process of evaluation of astrometric observable. The major difficulty is to analytically integrate a set of non linear coupled differential equations which allow to trace back the star positions and which include, a priori by definition, the background property of a curved space-time. At present, we can integrate the equations of the model numerically. A semi-analytical solution has been discussed in de Felice \& Preti (2006). Indeed, whether analytical or numerical, the solution of those equations contains "globally" all relativistic perturbations to a photon moving along its trajectory. A boundary conditions fixed by the astrometric observables as a function of analytical relativistic description of the satellite solves the Cauchy problem and allows a unique prediction for the stellar location (Bini et al. 2003). From the theoretical point of view, RAMOD is complete and ready to be implemented in the end-to-end simulation of a Gaia mission, aimed to estimate the astrometric parameters of celestial objects from a well-defined set of measured quantities.

\section{The pM/RAMOD comparison model}

A first comparison between different approaches can be done by using the GREM definition of the observed stellar direction together with the light deflection terms computed in the pM approach. In the latter the hyperbolic character of field equations is preserved and the positions of the bodies are a priori functions of retarded time as in RAMOD. More generally, the proper stellar direction can be expressed "globally" as a sum of a set of relativistic astrometric parameters (RAPs) for the Gaia-like catalogue and can be directly compared with the general expression that will be soon derived by the RAMOD-like models. The astrometric parameters depend on which part of the Galaxy Gaia will be observing, as they simultaneously link all possible "astrometric" relativistic effects related to the light propagation. A complete set of RAPs have already been computed for stars in the solar vicinity (Crosta, 2003), i.e. the expressions for the aberration, barycentric direction, deflection, parallaxes and, proper motion parameters accurate up to a $0.1 \mu$ as $\left(\sim\left(v_{\text {planet }} / c\right)^{3}\right)$. Part of the relativistic effects could be induced by the adopted approximation scheme and relativistic coordinate transformations which utilize several expansions with respect to a small parameter $\epsilon \sim\left(v_{\text {planet }} / c\right)$. Future work will include the analysis of each term's significance in order to investigate whether all these terms are physically related to the stellar kinematical properties. Term by term comparisons using the final solution of the covariant approach of RAMOD, as well as the GREM formalism developed in the Gaia context, should provide a definite answer.

\section{References}

Bini, D., Crosta, M. T., De Felice, F., 2003,Class.Quantum Grav., 20, 4695-4706

Crosta, M. T., 2003, Methods of Relativistic Astrometry for the analysis of astrometric data in the gravitational field of the Solar System, CISAS-University of Padova

de Felice \& Clarke, 1990, Relativity on curved Manifolds, Cambridge University press

de Felice, F., Crosta, M. T., Vecchiato, A., Bucciarelli, B., Lattanzi, M. G., 2004, ApJ, 607(1), 580-595

de Felice, F., Vecchiato, A., Crosta, M. T., Bucciarelli, B., Lattanzi, M. G., 2006, ApJ, 653, $1552-1565$

de Felice, F., \& Preti, G., 2006, Class. Quantum Grav.,23, 5467-5476

Klioner,, S.,\& Kopejkin, S. M.,1992, AJ, 104 (2), 897

Klioner, S., 2003, AJ, 125, 1580

Kopeikin, S., \& Schäfer,G., 1999, Phy.Rev.D, 60, 124002

Kopeikin, S. M., \& Mashhoon, B., 2002, Phy.Rev.D, 65, 064025 